\title{
Palm oil: features and applications
}

\begin{abstract}
Palm fruits are the source of two distinct but related vegetable oils, namely palm oil and palm kernel oil. Palm oil has many food and industrial applications. Ever-increasing demands for palm oil have substantially impacted its industry, creating some environmental concerns. Both types of oil are high in saturated fatty acids with potential cardiovascular risks. Several attempts have been made to reduce undesirable health and environmental impacts. However, additional research and development activities are needed to meet the concerns of the medical professionals and environmental activists.
\end{abstract}

Keyword: Palm oil; Palm fruit; Industrial application 